\title{
The BAFF/APRIL system in SLE pathogenesis.
}

Fabien B Vincent ${ }^{1}$, Eric F Morand ${ }^{2}$, Pascal Schneider ${ }^{3}$ and Fabienne Mackay ${ }^{1}$

${ }^{1}$ Department of Immunology, Monash University, Central Clinical School, Alfred Medical Research and Education Precinct (AMREP), 89 Commercial Road, Melbourne, Victoria 3004, Australia

${ }^{2}$ Monash University Centre for Inflammatory Diseases, Southern Clinical School, Monash Medical Centre, 246 Clayton Road, Clayton, Victoria, 3168, Australia

${ }^{3}$ Department of Biochemistry, University of Lausanne, Boveresses 155, 1066 Epalinges, Switzerland

\begin{abstract}
Systemic lupus erythematosus (SLE) is characterized by multisystem immune-mediated injury in the setting of autoimmunity to nuclear antigens. The clinical heterogeneity of SLE, the absence of universally agreed clinical trial end points, and the paucity of validated therapeutic targets have, historically, contributed to a lack of novel treatments for SLE. However, in 2011, a therapeutic monoclonal antibody that neutralizes the cytokine TNF ligand superfamily member 13B (also known as B-cell-activating factor of the TNF family [BAFF]), belimumab, became the first targeted therapy for SLE to have efficacy in a randomized clinical trial. Because of its specificity, the efficacy of belimumab provides an opportunity to increase understanding of SLE pathophysiology. Although belimumab depletes B cells, this effect is not as powerful as that of other B-cell-directed therapies that have not been proven efficacious in randomized clinical trials. In this article, therefore, we review results suggesting that neutralizing BAFF can have effects on the immune system other than depletion of B cells. We also identify aspects of the BAFF system for which data in relation to SLE are still missing, and we suggest studies to investigate the pathogenesis of SLE and ways to refine anti-BAFF therapies. The role of a related cytokine, TNF ligand superfamily member 13 (also known as a proliferation-inducing ligand [APRIL]) in SLE is much less well understood, and hence this review focuses on BAFF.
\end{abstract}




\section{Keywords}

A proliferation-inducing ligand (APRIL); autoimmunity; B cell activating factor of the tumour necrosis factor (TNF) family (BAFF); innate immunity; interferon (IFN); systemic lupus erythematosus (SLE).

\section{Review criteria}

We performed a PubMed search for relevant articles from 1999-2013, using key words, including "systemic lupus erythematosus", "BAFF", "BLyS", "THANK", "TALL-1", "zTNF4", “TNFSF13B”, "APRIL”, “TNFSF13”, "hetero-trimer”, "TACI”, "BAFF-R", "BCMA", "Nogo-66 receptor", "B cell”, “T cell”, "natural killer", “dendritic cell”, “neutrophils", “interferon”, “toll-like receptor", "Belimumab”, “GSK1550188”, "HGS1006”, "Blisibimod", “AMG623”, “A-623”, “Tabalumab”, and "LY2127399”. Articles in English were reviewed by title and abstract and selected if deemed important and relevant.

\section{Key points}

- TNF ligand superfamily member 13B (also known as B cell-activating factor of the TNF family $[\mathrm{BAFF}]$ ) and TNF ligand superfamily member 13 (also known as a proliferationinducing ligand [APRIL]) are important modulators of autoimmunity

- Data indicate that alteration of the BAFF/APRIL system affects the capacity of the innate immune system to regulate B-cell activation

- BAFF and type I interferons function together in systemic lupus erythematosus (SLE) pathogenesis in both a Toll-like receptor-dependent and independent manner

- Defining the clinical manifestations of disease related to alterations of the BAFF/APRIL system might help to stratify patients with SLE into subgroups that are more likely to benefit from anti-BAFF treatment

- Differences in the molecular forms of BAFF might affect the efficacy of BAFF-specific therapies 


\section{Introduction}

Systemic lupus erythematosus (SLE) is an idiopathic, systemic autoimmune disease. ${ }^{1}$ Although the cause of this disease is unclear, research over the past two decades has provided new insights into its pathogenesis. One breakthrough was the discovery, in 1999, of a crucial B-cell survival factor, TNF ligand super family member 13B (also known as B cell-activating factor of the TNF family [BAFF], B-lymphocyte stimulator [BLyS], zTNF4, THANK and TALL1), ${ }^{2}$ which has an important role in autoimmunity, and in particular in SLE pathogenesis. $^{3,4}$ In 2011, belimumab, a mono clonal antibody targeting human BAFF, was shown in randomized clinical trials to be efficacious in a subset of patients with SLE and has now become the first approved targeted therapy for SLE. 5,6 As a consequence, clinical inhibition of BAFF has generated substantial interest and similar biologic agents are being tested in clinical trials. The efficacy of a specific anti-BAFF treatment demonstrated that SLE is not exclusively a T-cell mediated disease and has renewed the scientific community's interest in the pathogenesis of SLE.

Clinical trials and post hoc analysis of data obtained from belimumab treated patients with SLE have pro vided useful information about BAFF and a related cytokine, TNF ligand superfamily member 13 (also known as a proliferation inducing ligand [APRIL]). Data suggest that mechanisms other than $\mathrm{B}$ cell depletion are affected during responses to treatment with belimumab. In addition, the deregulation of BAFF and APRIL is found in specific subsets of patients with SLE. ${ }^{7}$ The 'BAFF/APRIL system' and the innate immune system coordinate in regulating the innate activation of B cells, and seem to be defective in a mouse model of SLE in which BAFF is overexpressed. ${ }^{8}$ Several receptors and various biochemical forms of the ligands exist in the BAFF/APRIL system, ${ }^{9-11}$ but further work is required to elucidate their respective contributions to the pathogenesis and phenotype of SLE. In this article, we review the BAFF/APRIL system and highlight new discoveries that are relevant to the improvement of SLE management and that might lead to therapeutic innovation. In light of an ongoing debate about the role of APRIL in SLE, we have predominantly focused this Review on BAFF.

\section{The BAFF/APRIL system}

Two ligands, BAFF and APRIL, and three receptors, TNF receptor superfamily member 13C (also known as BAFF receptor [BAFFR] or BLyS receptor 3 [BR3]), TNF receptor superfamily member 17 (also known as B-cell maturation antigen [BCMA]) and TNF 
receptor superfamily member 13B (also known as transmembrane activator and cyclophilin ligand interactor [TACI]) form the backbone of the BAFF/APRIL system. BAFF and APRIL can both interact with BCMA and TACI, whereas BAFF is the sole ligand for BAFFR (reviewed previously ${ }^{12}$; Figure 1). BAFFR is essential for both survival and maturation of immature B cells, whereas TACI is critical for T-cell-independent responses of B cells to type I and type II antigens, negative regulation of the B-cell compartment and class-switch recombination of $\mathrm{B}$ cells (reviewed previously ${ }^{13}$ ). BCMA is expressed by plasmablasts and plasma cells, and promotes plasma cell survival.

BAFF and APRIL are produced by myeloid cells, pre dominantly by macrophages, neutrophils and dendritic cells (DCs), and also by radiation-resistant stromal cells. ${ }^{14}$ Lymphoid cells, including B cells and activated T cells, can also produce BAFF and APRIL. Finally, Toll like receptor 9 (TLR9)-activated plasmacytoid DCs and IL2-activated natural killer cells also produce BAFF (reviewed previously ${ }^{12}$ ).

BAFF and APRIL are type II transmembrane pro teins. ${ }^{3,15,16}$ BAFF can also be processed into a soluble cytokine after cleavage at a furin protease site, ${ }^{3,15}$ whereas APRIL is mainly produced in a soluble form. ${ }^{16,17}$ Two exceptions, which are membrane bound, are the APRIL $\delta$ isoform, first identified in malignant B-cell tumours, ${ }^{18}$ and TWEPRIL, a hybrid protein of APRIL and TNF ligand superfamily member 12 (also known as TNF related weak inducer of apoptosis [TWEAK]). ${ }^{19}$ The receptor-binding domain of BAFF is trimeric; ${ }^{20}$ however, 20 BAFF trimers can assemble into a BAFF 60mer. ${ }^{10,11}$ Furthermore, BAFF and APRIL can assemble as BAFF - APRIL heterotrimers of undefined stoichiometries, which have been detected in the serum of patients with rheumatic diseases (Figure 2). Recombinant BAFFAPRIL heterotrimers are biologically active, albeit less than BAFF or APRIL homotrimers. $^{21,22}$ Hetero-trimers of various compositions might be differentially susceptible to BAFF-targeting therapies.

Mice expressing BAFF with a mutated furin cleavage site have a phenotype similar to Baff-/(Tnfsf13b-/-) mice, suggesting that membrane-bound BAFF cannot substitute for soluble BAFF. ${ }^{23}$ However, when treated with soluble BAFF, B cells from the BAFF furin-mutant mice became more similar to B cells from wildtype mice than to B cells from Baff-/- mice that were also treated with soluble BAFF. ${ }^{23}$ These results suggest a twostep process in BAFFmediated B-cell maturation. First, soluble BAFF promotes B-cell survival by activating BAFFR, and then the B-cell phenotype is modulated by membrane-bound BAFF signals, possibly by activating TACI. $^{23}$ 
Human BAFF is a 285 amino acid-long protein with two potential $N$-glycosylation sites. ${ }^{24}$ Because some enzyme-linked immunosorbent assays (ELISAs) detect only unglycosylated BAFF, whereas other ELISAs detect both unglycosylated and glycosylated BAFF, posttranslational $\mathrm{N}$-glycosylation status could explain the failure to consistently associate disease activity with serum BAFF concentrations. ${ }^{24}$ Alternative splice variants of BAFF, particularly $\triangle \mathrm{BAFF}$, might have an important role in SLE pathogenesis. ${ }^{25-28}$ Mouse $\triangle \mathrm{BAFF}$ decreases the bioactivity and release of full-length BAFF by associating with BAFF and forming inactive hetero trimers. ${ }^{26}$ In mice, BAFF and $\triangle \mathrm{BAFF}$ can both modulate B1 B cells. ${ }^{25,26}$ BAFF $\varphi$ is a nonfunctional isoform identified in human cell lines. ${ }^{26}$ Although currently unknown, it is possible that anti-BAFF biologic agents might differ in their ability to bind and neutralize the various human BAFF isoforms or glycosylation variants.

\section{The BAFF/APRIL system in SLE}

BAFF is necessary for B-cell maturation and survival. Mice genetically deficient in BAFF lack mature B cells and are immunodeficient, ${ }^{29}$ whereas mice that over produce BAFF have high numbers of mature B cells and antibodies, including autoantibodies, and develop an autoimmune disease similar to SLE in humans. ${ }^{2}$ Together with the observation that BAFF blockade decreased symptoms of SLE in mouse models, ${ }^{30}$ these findings promoted BAFF as a therapeutic target for the treatment of patients with SLE.

APRIL is important for antibody class-switching and plasma-cell survival (reviewed previously ${ }^{13}$ ). APRIL overexpressing transgenic mice develop B1 B-cell neoplasia, but do not develop SLE-like pathology. ${ }^{31}$ Selective APRIL blockade can delay the development of disease in a lupus-prone mouse model (NZB/W F1 mice). ${ }^{32}$ Using NZM.April-/(NZM.Tnfsf13-/-) mice, Jacob et al. ${ }^{33}$ showed that, in this model, APRIL was not necessary for SLE pathogenesis. Although NZM.Baff-/-April-/- (NZM. Tnfsf13b-/-Tnfsf13-/-) mice had fewer bone-marrow plasma cells and autoantibodies than NZM.Baff-/- (NZM.Tnfsf13b/-) mice, both strains had mild kidney immunopathology, ${ }^{33}$ suggesting that blocking both BAFF and APRIL in patients with SLE might not be beneficial and could even increase the risk of toxicity. Further supporting this idea, a phase II/III clinical trial of the chimeric recombinant fusion protein atacicept (combining TACI with an immunoglobulin constant region), which neutralizes BAFF, APRIL and BAFF-APRIL hetero-trimers in patients with active lupus nephritis, was stopped because of serum IgG depletion and an increased rate of infections. ${ }^{34}$ 


\section{Belimumab}

Belimumab is a fully human recombinant monoclonal $\operatorname{IgG} \lambda$ antibody that impairs Bcell survival by targeting soluble, not membrane-bound, human BAFF. ${ }^{35}$ The addition of belimumab to standard therapy has been studied in two multi-centre, double-blind, placebocontrolled, randomized phase III clinical trials in patients with SLE, cumulatively examining 1,684 patients for up to 76 weeks. ${ }^{5,6}$ Various scoring systems are used to evaluate disease activity in human SLE clinical trials, such as SLE disease activity index (SLEDAI), British isles lupus assessment group (BILAG) or SLE responder index (SRI). ${ }^{36-38}$ These composite scores of clinical and laboratory features enable scaled assessment of disease activity at a single time point. Patients with active SLE who tested positive for SLE-associated antibodies benefited from belimumab compared with placebo with respect to disease activity (SRI) and

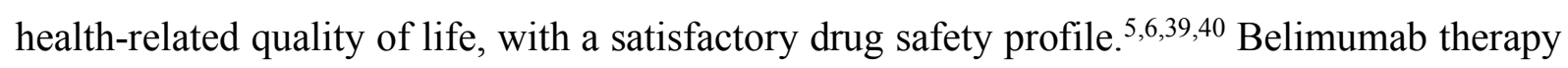
was associated with reduced autoantibody levels and normalized low complement levels, ${ }^{41}$ and in post hoc analysis, patients with high disease activity (Safety Of Oestrogens In Lupus Erythematosus National Assessment [SELENA] SLEDAI), high anti-double-stranded DNA antibody and low complement levels at baseline had superior treatment responses to belimumab. ${ }^{42}$ Belimumab therapy also reduced the number of circulating naive $\mathrm{B}$ cells, activated B cells and plasma cells, but did not reduce the number of circulating memory B cells or $\mathrm{T}$ cells. ${ }^{41}$ No effects on antibody responses to previous pneumococcal, tetanus or influenza immunizations were detected after 1 year of treatment, consistent with a preserved memory B-cell compartment. ${ }^{43}$

\section{BAFF and APRIL as biomarkers of SLE}

In comparison with serum from healthy individuals, serum concentrations of BAFF and APRIL are higher in patients with autoimmune diseases, such as SLE, primary Sjögren's syndrome and rheumatoid arthritis (reviewed previously ${ }^{13}$ ). Whether serum BAFF or APRIL can be used as a biomarker in SLE has been a matter of debate over the past decade, with some studies reporting correlations between serum BAFF and APRIL concentrations and overall disease activity, ${ }^{44-47}$ whereas others found no such correlations. ${ }^{41,47-50}$ Petri et al. ${ }^{51}$ showed, using multivariate analysis, that elevated baseline serum BAFF concentration $(\geq 2$ $\mathrm{ng} / \mathrm{ml}$ ) was predictive of moderate-to-severe SLE flares in patients receiving the standard therapy, prednisone with antimalarial (hydroxychloroquine) or immunosuppressive drugs 
(methotrexate, mycophenolate mofetil and azathioprine). This association is supported by another study, in which a high serum BAFF concentration was associated with flares of SLE disease activity (assessed by the BILAG score) after rituximab treatment.52 However, in our study, ${ }^{7}$ among others, no significant association between serum BAFF concentration and disease activity (assessed using SLEDAI2k) was found. Moreover, in the belimumab phase III clinical trials, baseline serum BAFF concentration was not found to be predictive of outcome in either anti-BAFF or control-treated patients. ${ }^{41}$ These discrepancies might be caused by differences in assay sensitivity, disease activity scores or study populations. However, serum BAFF and APRIL concentrations might not accurately reflect total BAFF production or, in the case of APRIL (which binds proteoglycans), the concentration of the active cytokine in tissues. Urinary excretion of cytokines, for example, might reduce serum concentration in patients with renal disease, suggesting that the measurement of BAFF and APRIL in urine should be investigated by SLE researchers. ${ }^{53}$

Perhaps the failure to reliably show an association between disease activity and the concentration of a cytokine known to be involved in the pathogenesis of SLE means that the measurement of disease activity requires reexamination. Moreover, given that post hoc analysis of clinical trial data revealed that BAFF inhibition was most effective in patients with active disease and high serum autoantibody titres, ${ }^{42}$ this subset of SLE patients might be best suited for analysis of clinical associations of BAFF and APRIL expression. We could, therefore, study SLE patient subsets, defined according to specific clinical or immunological phenotypes.

\section{SLE clinical subsets}

Some studies measuring cytokines in patients with SLE have focused on SLE subsets rather than over all disease activity measured with composite indices of disease activity. ${ }^{54-60} \mathrm{We}$ showed that serum BAFF and APRIL concentrations, although not correlating with a composite measure of disease activity (SLEDAI2k), were increased and decreased, respectively, in sub sets of patients with SLE who also had renal or central nervous system (CNS) pathology. ${ }^{7}$ These data are sup ported by findings from mouse studies, in which separate pathways that regulate autoimmunity, inflammation and renal damage led to differentiated phenotypes of disease. ${ }^{61,62}$ Combining different genetic loci such as Sle3 and Sle5, with or without Sle1, leads to differentiated phenotypes in lupus-prone mice. ${ }^{62}$ If distinct 
immunological pathways are activated in humans with different disease manifestations, then general biomarkers for SLE might not exist. Some subsets of patients with SLE might, however, benefit from therapies targeting these pathways. The identification of reliable biomarkers for these subsets would be useful as a tool for the selection of therapy. Serum $\mathrm{BAFF}$, for example, is increased in patients with CNS or renal pathology, therefore, these patients might benefit from anti-BAFF therapy. ${ }^{7}$ Although patients with severe CNS manifestations or severe lupus nephritis were excluded from two phase III clinical trials of belimumab, ${ }^{5,6}$ post hoc pooled analysis suggested that belimumab might be an effective treatment for patients with SLE and renal involvement.63 Also, in the subset of SLE patients without renal disease activity at baseline, those treated with belimumab had less renal involvement than patients given placebo. ${ }^{64}$ Further research is needed to investigate the accuracy of using serum BAFF and APRIL measurements to predict SLE phenotype, and therapeutic responses in patients within the different subgroups.

\section{Ethnicity and polymorphisms}

Substantial ethnic variation in the prevalence and characteristics of SLE exists. ${ }^{65,66}$ Asian, Indigenous Australian and African patients with SLE suffer from more severe disease compared with white people. ${ }^{7,65-68}$ In one report, serum BAFF or APRIL concentration was not associated with Asian ethnicity;7 however, African American patients with SLE had higher serum BAFF concentrations than white American patients with SLE, although not significantly after multivariate analysis. ${ }^{69}$ By contrast, increased serum BAFF concentration was associated with an increase in the SLEDAI in white American patients, but not in African American patients. ${ }^{69}$ Further research is needed to understand how particular ethnic subgroups respond to anti-BAFF therapies.

Future research could focus on BAFF and APRIL single nucleotide polymorphisms (SNPs). Several SNPs have been identified in the promoter, coding and untranslated regions of TNFSF13B (the human BAFF gene), but in most studies no significant association with SLE susceptibility has been found. ${ }^{70,71}$ Only one study reported an association (in Egyptian patients with SLE) between disease susceptibility and $871 \mathrm{C}>\mathrm{T}$ and $2701 \mathrm{~T}>\mathrm{A}$ SNPs in the BAFF promoter region. ${ }^{72}$ TNFSF $13 B$ pro moter SNPs were not significantly associated with symptoms of SLE. Monocytes from healthy individuals with the $871 \mathrm{~T}$ allele had a significant increase in BAFF mRNA compared with monocytes from healthy individuals without the $871 \mathrm{~T}$ allele. ${ }^{70}$ 
SNPs have also been found in TNFSF13 (the human APRIL gene), including Gly67Arg, which was significantly associated with SLE susceptibility in Japanese patients. ${ }^{73}$ Another study showed a possible association between Gly67Arg and SLE susceptibility in African American and Hispanic people, but not in white American people. ${ }^{74}$ Additionally, the c.199Ac.287G (67Arg96Ser) SNP was reported to be a protective haplotype whereas the c.199Gc.287A (67Gly96Asn) haplotype was associated with disease susceptibility in Japanese patients with SLE. ${ }^{75}$ In line with its protective effect, the protective haplotype 67Arg96Ser decreased soluble APRIL secretion in transfection experiments. ${ }^{76}$

\section{BAFF and APRIL receptors in SLE}

Exacerbation of disease in lupus-prone BCMA-deficient mice (MRLFaslpr/J.Tnfrsf17-/- and Nba2.Tnfrsf17-/- mice), suggests that BCMA has a direct or indirect negative regulatory role. ${ }^{77}$ In these mouse models, exacerbation of disease might occur by abnormal signalling of BAFF or APRIL through TACI or BAFFR in the absence of BCMA. Compared with wildtype and single-mutant control strains, BCMA-deficient lupus prone mice have more mature B cells and plasma cells in secondary lymphoid organs, more serum autoantibodies and BAFF, and more immune complex deposition in the kidneys. ${ }^{77} \mathrm{An}$ increase in the number of BAFF producing cells, such as DCs and macrophages, was also detected in these mice. ${ }^{77}$ BCMA signalling might have an indirect regulatory role in the homeostasis of BAFF producing cells, because BCMA was not expressed by splenic DCs in all mouse strains in this study (MRL Faslpr/J, Tnfrsf17-/-, MRLFaslpr/J.Tnfrsf17-/and wild type mice). ${ }^{77}$ Jacob et al. ${ }^{78}$ showed that the deletion of BAFFR alone, which leads to profound B-cell depletion, or of BCMA or TACI alone, did not protect against SLE-like disease in NZM lupus-prone mice. These data suggest that, unlike BAFF-overexpressing transgenic mice, ${ }^{79} \mathrm{~T}$ cells might have an important role in the pathogenesis of NZM lupusprone mice. ${ }^{78}$

the absence of BCMA. Compared with wildtype and single-mutant control strains, BCMAdeficient lupus prone mice have more mature B cells and plasma cells in secondary lymphoid organs, more serum autoantibodies and BAFF, and more immune complex deposition in the kidneys. ${ }^{77}$ An increase in the number of BAFF producing cells, such as DCs and macrophages, was also detected in these mice. ${ }^{77}$ BCMA signalling might have an indirect regulatory role in the homeostasis of BAFF producing cells, because BCMA was not expressed by splenic DCs in all mouse strains in this study (MRL Faslpr/J, Tnfrsf17-/-, MRL 
Faslpr/J.Tnfrsf17-/- and wild type mice). ${ }^{77}$ Jacob et al. ${ }^{78}$ showed that the deletion of BAFFR alone, which leads to profound B-cell depletion, or of BCMA or TACI alone, did not protect against SLE-like disease in NZM lupus-prone mice. These data suggest that, unlike BAFFoverexpressing transgenic mice, ${ }^{79} \mathrm{~T}$ cells might have an important role in the pathogenesis of NZM lupus-prone mice. ${ }^{78}$

\section{BAFF, TLR cross-talk and IFN signatures}

Type I interferons, and particularly IFNa, are thought to have a major role in SLE pathogenesis. SLE is characterized by increased expression of IFN $\alpha$-induced genes, referred to as the IFN $\alpha$ signature, in peripheral blood mononuclear cells and in target organs, such as the kidneys. ${ }^{54,82,83}$ In some studies, serum IFNa concentrations and IFN-induced genes positively correlate with SLE disease activity (measured by SLEDAI). ${ }^{82,84}$ IFNG expression is also increased in SLE, ${ }^{60}$ and higher serum IFN $\gamma$ concentrations have been reported in patients with SLE compared with healthy individuals. ${ }^{85}$ Plasmacytoid DCs are the major source of IFN $\alpha$ produced in response to viruses and bacteria (reviewed previously ${ }^{86}$ ). Activation of endosomal TLR7 and TLR9 by their respective agonists, single-stranded RNA and unmethylated CpG-rich DNA, transduces signals through myeloid differentiation primary response protein MyD88 (MyD88) and upregulates IFNa expression. In SLE, antibodynucleic acid immune complexes, for example RNA or DNA from necrotic or apoptotic cells, ${ }^{87}$ are suggested to be bound by immunoglobulin $\gamma \mathrm{Fc}$ region receptor IIa (FcyRIIa) and internalized by plasmacytoid DCs in the form of autoimmune complexes, subsequently activating endosomal TLRs and upregulating IFN $\alpha$ production (Figure 3). ${ }^{86,88}$

Several studies indicate that interferons induce BAFF expression: IFN $\alpha$ upregulates BAFF expression by mouse macrophages, ${ }^{89}$ IFN $\alpha$ and IFN $\gamma$ both upregulate BAFF and APRIL expression by human DCs $;{ }^{90}$ and IFN $\alpha$ and IFN $\gamma$ can upregulate BAFF expression by human monocytes. ${ }^{90}$ In response to IFN $\gamma$, more BAFF was produced by monocytes from patients with SLE than by monocytes from healthy donors. ${ }^{91}$ In a phase Ia clinical trial of patients with SLE treated with an anti-IFN $\alpha$ monoclonal antibody, BAFF mRNA expression in whole blood was suppressed. ${ }^{92}$ Strikingly, in NZM lupus-prone mice, BAFF deficiency prevented glomerulonephritis and clinical disease in response to IFN $\alpha$ treatment. ${ }^{93}$ The activity of IFN $\alpha$ in serum from patients with SLE positively correlated with serum BAFF concentrations. ${ }^{69}$ Moreover, we demonstrated a regulatory feedback loop between TLR7 or TLR9 and TACI in BAFF transgenic mice, ${ }^{79}$ and cooperation between TACI and TLR 
signalling in Fas-induced apoptosis, a mechanism that is defective in these mice (Box 1). ${ }^{8}$ Collectively, these mouse and human studies of SLE pathogenesis suggest that the roles of BAFF and IFN $\alpha$ are closely linked (Figure 3). One study showed that, through an IFN $\alpha-$ dependent pathway, monocytes from healthy individuals can differentiate into DCs when cultured with serum from patients with SLE (SLEDCs), and this induction of DC differentiation also correlated with the SLE disease activity (measured by SLEDAI). ${ }^{94}$ SLEDCs can induce B-cell proliferation and plasma blast differentiation..$^{95}$ Interestingly, DCs differentiated from monocytes, from healthy individuals, and cultured with IFN $\alpha$ plus granulocyte macrophage colony stimulating factor (IFNDCs), phenotypically differ from SLEDCs. One of the main differences is that IgG secretion was enhanced (dependent on BAFF) by SLEDCs but not IFNDCs. ${ }^{95}$

\section{Therapeutic innovation}

Although known anti-BAFF biologic agents bind to soluble or membrane-bound BAFF, the specificity for homotrimer, hetero-trimer and 60-mer forms is unclear. Belimumab targets only soluble human BAFF. ${ }^{35}$ Tabalumab (also known as LY2127399), a fully-human IgG4 monoclonal antibody, neutralizes both soluble and membrane-bound human BAFF. ${ }^{96}$ Blisibimod (also known as A623) is a fusion polypeptide protein that targets human BAFF and binds to both soluble and membrane-bound BAFF in mouse models of SLE and rheumatoid arthritis. ${ }^{97}$

Major differences between BAFF trimer and 60mer forms have been investigated using mouse models; however, in humans, these forms of BAFF have not been studied in detail and the 60-mer has not been described. In primary B cells isolated from mice, TACI is activated by the BAFF 60-mer, but not the BAFF trimer. ${ }^{20}$ TACI interacts with MyD88, a signalling adaptor critical for the development of BAFF-mediated autoimmunity in mice, ${ }^{79}$ suggesting that TACI, and hence the BAFF 60-mer, might be important in regulating autoimmune disease. One of the major unanswered questions is whether human BAFF exists in both trimer and 60-mer forms, and what functions the different forms might have in disease. Indeed, if the BAFF 60-mer is the active form in humans, active-form-specific anti-BAFF therapies might be developed by neutralizing only the active BAFF 60 -mer, or by therapeutically disrupting the active 60-mer into the less active trimer (Figure 2). The BAFF 60-mer is not an established molecular form of BAFF in vivo and has yet to be characterized in mice and humans. 
If findings on the reduced bioactivity of recombinant BAFF-APRIL hetero-trimers apply to the native hetero trimer, hetero-trimer formation could have a role in determining BAFF or APRIL activity in vivo. Analysis performed on a small cohort of 36 patients with SLE showed a nonsignificant trend towards a positive correlation between serum BAFF-APRIL heterotrimer con centration and disease activity (measured by SLEDAI). ${ }^{21}$ If supported by larger longitudinal, prospective studies, these data could be interpreted to mean either that heterotrimer formation drives specific pathogenic signals or that it reflects the action of a negative feedback loop without which the pathology is more severe (Figure 2). Whether hetero-trimer formation occurs through de novo assembly of monomers in a cell producing both BAFF and APRIL, or whether homotrimers equilibrate into hetero-trimers after synthesis and release is not known. However, hetero-trimers can be predicted not to form 60mers. BAFF contains a loop sequence (the 'flap') that protrudes out of the monomer and hooks to the flap of BAFF in adjacent trimers. ${ }^{10}$ Size analyses of recombinant BAFF 60 -mers, showed some BAFF trimers in addition to the 60-mer, but no 6-mer or 9-mer (etc.) intermediates, ${ }^{11}$ suggesting that flapflap interactions are weak and are probably only important when they act cooperatively in the 60-mer. APRIL has no flap sequence ${ }^{98}$ and, therefore, theoretically cannot be incorporated into a BAFF 60mer; if APRIL were incorporated it would most likely lead to 60mer dissociation. A better understanding of the relative proportions and activity of BAFFAPRIL homotrimers and hetero-trimers might redirect therapeutic targeting of the BAFF/APRIL system.

\section{Conclusion}

The approval of BAFF inhibition as the first targeted therapy for SLE is a major advance in the treatment of this disease, and confirms a role for BAFF in the pathogenesis of SLE. However, the diversity of SLE manifestations and the monetary cost of biologic therapy might restrict the generalized use of anti-BAFF biologic agents in the treatment of SLE. The role of the BAFF/APRIL system in the pathogenesis of SLE needs to be more completely understood in order to improve stratification of patients with SLE for effective anti-BAFF therapy. Studies of clinical subsets of patients who overexpress BAFF, but who were previously excluded from clinical trials, are particularly needed. Novel data suggest the potential for various approaches to target the BAFF/APRIL system in patients with SLE, such as targeting BAFF 60mers, hetero-trimers or novel receptors. Further work on the biology of the BAFF/APRIL system, and its connection to type I interferons and innate 
immunity (Box 2), together with rapid translation to clinical validation, is required. The role of current and future BAFF/APRIL system targeting therapies in SLE will be determined by careful application of experimental and clinical approaches.

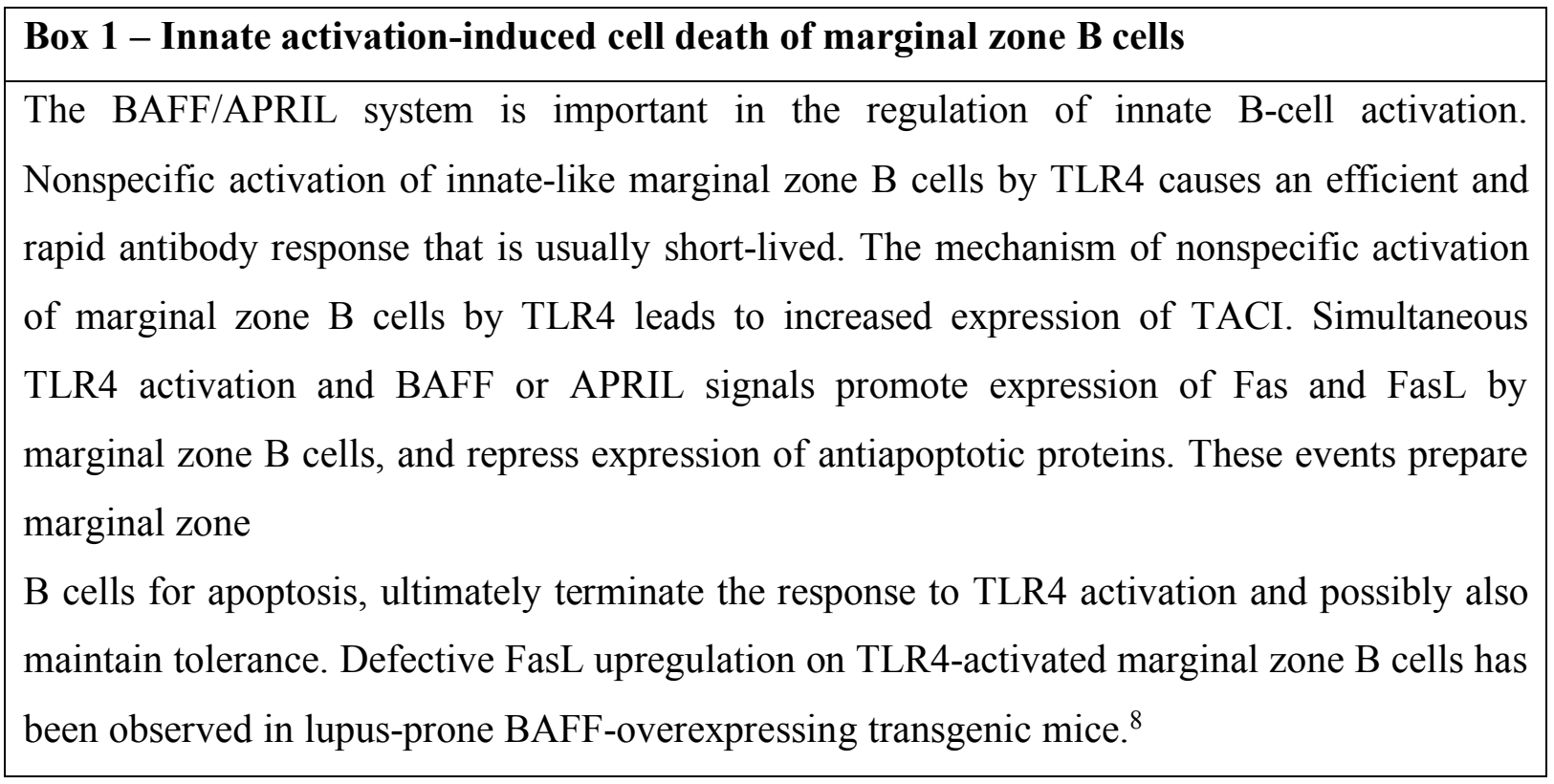

\section{Box 2 - BAFF and immunity}

- Studies showed that BAFF (and APRIL) is involved in splenic neutrophil activation of marginal zone B cells, promotion of plasma cell differentiation and increased immunoglobulin production ${ }^{99}$

- Cytoplasmic anti-neutrophil antibodies stimulate BAFF production by neutrophils leading to enhanced B-cell survival ${ }^{100}$

- Neutrophils infiltrating the joints of patients with rheumatoid arthritis release BAFF after TNF activation ${ }^{101}$

- Human NK cells produce BAFF when stimulated with IL-2 $2^{102}$

- An in vitro study of mouse splenocytes showed that soluble BAFF can indirectly enhance NK-cell activity through upregulation of IL-2 and IFN- $\gamma$ production by CD4+ T cells ${ }^{103}$ 


\section{References}

1. Tsokos, G. C. Systemic lupus erythematosus. N. Eng. J. Med. 365, 2110-2121 (2011).

2. Mackay, F. et al. Mice transgenic for BAFF develop lymphocytic disorders along with autoimmune manifestations. J. Exp. Med. 190, 1697-1710 (1999).

3. Schneider, P. et al. BAFF, a novel ligand of the tumor necrosis factor family, stimulates B cell growth. J. Exp. Med. 189, 1747-1756 (1999).

4. Batten, M. et al. BAFF mediates survival of peripheral immature B lymphocytes. J. Exp. Med. 192, 1453-1466 (2000).

5. Navarra, S. V. et al. Efficacy and safety of belimumab in patients with active systemic lupus erythematosus: a randomised, placebo- controlled, phase 3 trial. Lancet 377, 721-731 (2011).

6. Furie, R. et al. A phase 3, randomized, placebo- controlled study of belimumab, a monoclonal antibody that inhibits BLyS, in patients with systemic lupus erythematosus. Arthritis Rheum. 63, 3918-3930 (2011).

7. Vincent, F. B., Northcott, M., Hoi, A., Mackay, F. \& Morand, E. F. Association of serum B cell activating factor from the tumour necrosis factor family (BAFF) and a proliferationinducing ligand (APRIL) with central nervous system and renal disease in systemic lupus erythematosus. Lupus 22, 873-884 (2013).

8. Figgett, W. A. et al. The TACI receptor regulates T-cell-independent marginal zone B cell responses through innate activation-induced cell death. Immunity 39, 573-583 (2013).

9. Zhang, L. et al. Identification of BLyS (B lymphocyte stimulator), a non-myelin- associated protein, as a functional ligand for Nogo-66 receptor. J. Neurosci. 29, 6348-6352 (2009).

10. Liu, Y. et al. Crystal structure of sTALL-1 reveals a virus-like assembly of TNF family ligands. Cell 108, 383-394 (2002).

11. Cachero, T. G. et al. Formation of virus-like clusters is an intrinsic property of the tumor necrosis factor family member BAFF (B cell activating factor). Biochemistry 45, 2006-2013 (2006).

12. Vincent, F. B., Morand, E. F. \& Mackay, F. BAFF and innate immunity: new therapeutic targets for systemic lupus erythematosus. Immunol. Cell Biol. 90, 293-303 (2012).

13. Vincent, F. B., Saulep-Easton, D., Figgett, W. A., Fairfax, K. A. \& Mackay, F. The BAFF/APRIL system: Emerging functions beyond B cell biology and autoimmunity. Cytokine Growth Factor Rev. 24, 203-215 (2013).

14. Gorelik, L. et al. Normal B cell homeostasis requires B cell activation factor production by radiation-resistant cells. J. Exp. Med. 198, 937-945 (2003).

15. Moore,P.A.etal.BLyS:memberofthetumor necrosis factor family and B lymphocyte stimulator. Science 285, 260-263 (1999). 
16. Hahne, M. et al. APRIL, a new ligand of the tumor necrosis factor family, stimulates tumor cell growth. J. Exp. Med. 188, 1185-1190 (1998).

17. López-Fraga, M., Fernandez, R., Albar, J. P. \& Hahne, M. Biologically active APRIL is secreted following intracellular processing in the Golgi apparatus by furin convertase. EMBO Rep. 2, 945-951 (2001).

18. Maia, S. et al. Aberrant expression of functional BAFF-system receptors by malignant B-cell precursors impacts leukemia cell survival. PLoS ONE 6, e20787 (2011).

19. Pradet-Balade, B. et al. An endogenous hybrid mRNA encodes TWE-PRIL, a functional cell surface TWEAK-APRIL fusion protein. EMBO J. 21, 5711-5720 (2002).

20. Bossen, C. et al. TACI, unlike BAFF-R, is solely activated by oligomeric BAFF and APRIL to support survival of activated B cells and plasmablasts. Blood 111, 1004-1012 (2008).

21. Dillon, S. R. et al. B-lymphocyte stimulator/a proliferation-inducing ligand heterotrimers are elevated in the sera of patients with autoimmune disease and are neutralized by atacicept and B-cell maturation antigen- immunoglobulin. Arthritis Res. Ther. 12, R48 (2010).

22. Roschke, V. et al. BLyS and APRIL form biologically active heterotrimers that are expressed in patients with systemic immune- based rheumatic diseases. J. Immunol. 169, 4314-4321 (2002).

23. Bossen, C. et al. Mutation of the BAFF furin cleavage site impairs B-cell homeostasis and antibody responses. E. J. Immunol. 41, 787-797 (2011).

24. Le Pottier, L. et al. New ELISA for B cell-activating factor. Clin. Chem. 55, 1843-1851 (2009).

25. Gavin, A. L. et al. $\triangle \mathrm{BAFF}$, a splice isoform of BAFF, opposes full-length BAFF activity in vivo in transgenic mouse models. J. Immunol. 175, 319-328 (2005).

26. Gavin, A. L., Aït-Azzouzene, D., Ware, C. F. \& Nemazee, D. $\triangle B A F F$, an alternate splice isoform that regulates receptor binding and biopresentation of the $\mathrm{B}$ cell survival cytokine, BAFF. J. Biol. Chem. 278, 38220-38228 (2003).

27. Krumbholz, M. et al. BAFF is produced by astrocytes and up-regulated in multiple sclerosis lesions and primary central nervous system lymphoma. J. Exp. Med. 201, 195-200 (2005).

28. Lopez De Padilla, C. M. et al. BAFF expression correlates with idiopathic inflammatory myopathy disease activity measures and autoantibodies. J. Rheumatol. 40, 294-302 (2013).

29. Gross, J. A. et al. TACI-Ig neutralizes molecules critical for B cell development and autoimmune disease. impaired B cell maturation in mice lacking BLyS. Immunity 15, 289 302 (2001).

30. Gross, J. A. et al. TACI and BCMA are receptors for a TNF homologue implicated in B-cell autoimmune disease. Nature 404, 995-999 (2000).

31. Mackay, F. \& Schneider, P. Cracking the BAFF code. Nat. Rev. Immunol. 9, 491-502 (2009). 
32. Huard, B., Tran, N. L., Benkhoucha, M., Manzin- Lorenzi, C. \& Santiago-Raber, M. L. Selective APRIL blockade delays systemic lupus erythematosus in mouse. PLoS ONE 7, e31837 (2012).

33. Jacob, C. O. et al. Dispensability of APRIL to the development of systemic lupus erythematosus in NZM 2328 mice. Arthritis Rheum. 64, 1610-1619 (2012).

34. Ginzler, E. M. et al. Atacicept in combination with MMF and corticosteroids in lupus nephritis: results of a prematurely terminated trial. Arthritis Res. Ther. 14, R33 (2012).

35. Baker, K. P. et al. Generation and characterization of LymphoStat-B, a human monoclonal antibody that antagonizes the bioactivities of B lymphocyte stimulator. Arthritis Rheum. 48, 3253-3265 (2003).

36. Bombardier, C., Gladman, D. D., Urowitz, M. B., Caron, D. \& Chang, C. H. Derivation of the SLEDAI. A disease activity index for lupus patients. The Committee on Prognosis Studies in SLE. Arthritis Rheum. 35, 630-640 (1992).

37. Hay, E. M. et al. The BILAG index: a reliable and valid instrument for measuring clinical disease activity in systemic lupus erythematosus. Q. J. Med. 86, 447-458 (1993).

38. Furie, R. A. et al. Novel evidence-based systemic lupus erythematosus responder index. Arthritis Rheum. 61, 1143-1151 (2009).

39. Strand, V. et al. Improvements in health-related quality of life with belimumab, a Blymphocyte stimulator-specific inhibitor, in patients with autoantibody-positive systemic lupus erythematosus from the randomised controlled BLISS trials. Ann. Rheum. Dis. http://dx.doi.org/ 10.1136/annrheumdis-2012-202865.

40. Wallace, D. J. et al. Safety profile of belimumab: pooled data from placebo-controlled phase 2 and 3 studies in patients with systemic lupus erythematosus. Lupus 22, 144-154 (2013).

41. Stohl, W. et al. Belimumab reduces autoantibodies, normalizes low complement levels, and reduces select B cell populations in patients with systemic lupus erythematosus. Arthritis Rheum. 64, 2328-2337 (2012).

42. van Vollenhoven, R. F. et al. Belimumab in the treatment of systemic lupus erythematosus: high disease activity predictors of response. Ann. Rheum. Dis. 71, 1343-1349 (2012).

43. Chatham, W. W. et al. Effect of belimumab on vaccine antigen antibodies to influenza, pneumococcal, and tetanus vaccines in patients with systemic lupus erythematosus in the BLISS-76 trial. J. Rheumatol. 39, 1632-1640 (2012).

44. Petri, M. et al. Association of plasma B lymphocyte stimulator levels and disease activity in systemic lupus erythematosus. Arthritis Rheum. 58, 2453-2459 (2008).

45. Hegazy, M., Darwish, H., Darweesh, H., El- Shehaby, A. \& Emad, Y. Raised serum level of APRIL in patients with systemic lupus erythematosus: correlations with disease activity indices. Clin. Immunol. 135, 118-124 (2010). 
46. Stohl, W. et al. Inverse association between circulating APRIL levels and serological and clinical disease activity in patients with systemic lupus erythematosus. Ann. Rheum. Dis. 63, 1096-1103 (2004).

47. Morel, J. et al. Serum levels of tumour necrosis factor family members a proliferationinducing ligand (APRIL) and B lymphocyte stimulator (BLyS) are inversely correlated in systemic lupus erythematosus. Ann. Rheum. Dis. 68, 997-1002 (2009).

48. Stohl, W. et al. B lymphocyte stimulator overexpression in patients with systemic lupus erythematosus: longitudinal observations. Arthritis Rheum. 48, 3475-3486 (2003).

49. Zhang, J. et al. Cutting edge: a role for B lymphocyte stimulator in systemic lupus erythematosus. J. Immunol. 166, 6-10 (2001).

50. Collins, C. E. et al. B lymphocyte stimulator (BLyS) isoforms in systemic lupus erythematosus: disease activity correlates better with blood leukocyte BLyS mRNA levels than with plasma BLyS protein levels. Arthritis Res. Ther. 8, R6 (2006).

51. Petri, M. A. et al. Baseline predictors of systemic lupus erythematosus flares: Data from the combined placebo groups in the Phase 3 belimumab trials. Arthritis Rheum. 65, 2143-2153 (2013).

52. Carter, L. M., Isenberg, D. A. \& Ehrenstein, M. R. Elevated serum B-cell activating factor (BAFF/ BLyS) is associated with rising anti-dsDNA antibody levels and flare following Bcell depletion therapy in systemic lupus erythematosus. Arthritis Rheum. 65, 2672-2679 (2013).

53. Cheema,

G. $\quad$ S., $\quad$ Roschke, $\quad$ V., Hilbert,

D. M.

\& Stohl, W. Elevated serum B lymphocyte stimulator levels in patients with systemic immune-based rheumatic diseases. Arthritis Rheum. 44, 1313-1319 (2001).

54. Baechler, E. C. et al. Interferon-inducible gene expression signature in peripheral blood cells of patients with severe lupus. Proc. Natl Acad. Sci. USA 100, 2610-2615 (2003).

55. Vincent, $\quad$ F. $\quad$ B., $\quad$ Northcott, $\quad$ M., $\quad$ Hoi, Mackay, F. \& Morand, E. F. Clinical associations of serum interleukin-17 in systemic lupus erythematosus. Arthritis Res. Ther. 15, R97 (2013).

56. Kirou, K. A. et al. Activation of the interferon- $\alpha$ pathway identifies a subgroup of systemic lupus erythematosus patients with distinct serologic features and active disease. Arthritis Rheum. 52, 1491-1503 (2005).

57. Feng, X. et al. Association of increased interferon-inducible gene expression with disease activity and lupus nephritis in patients with systemic lupus erythematosus. Arthritis Rheum. 54, 2951-2962 (2006). 
58. Landolt-Marticorena, C. et al. Lack of association between the interferon-alpha signature and longitudinal changes in disease activity in systemic lupus erythematosus. Ann. Rheum. Dis. 68, 1440-1446 (2009).

59. Bertsias, G. K., Salmon, J. E. \& Boumpas, D. T. Therapeutic opportunities in systemic lupus erythematosus: state of the art and prospects for the new decade. Ann. Rheum. Dis. 69, 16031611 (2010).

60. Rana, A. et al. Gene expression of cytokines (TNF- $\alpha$, IFN- $\gamma$ ), serum profiles of IL-17 and IL23 in paediatric systemic lupus erythematosus. Lupus 21, 1105-1112 (2012).

61. Vuyyuru, $\quad$ R., $\quad$ Mohan, $\quad$ C., $\quad$ Manser, $\quad$ T. \& Rahman, Z. S. The lupus susceptibility locus Sle1 breaches peripheral B cell tolerance at the antibody-forming cell and germinal center checkpoints. J. Immunol. 183, 5716-5727 (2009).

62. Liu, K. et al. Sle3 and Sle5 can independently couple with Sle1 to mediate severe lupus nephritis. Genes Immun. 8, 634-645 (2007).

63. Dooley, M. et al. Effect of belimumab treatment on renal outcomes: results from the phase 3 belimumab clinical trials in patients with SLE. Lupus 22, 63-72 (2013).

64. Manzi, S. et al. Effects of belimumab, a B lymphocyte stimulator-specific inhibitor, on disease activity across multiple organ domains in patients with systemic lupus erythematosus: combined results from two phase III trials. Ann. Rheum. Dis. 71, 1833-1838 (2012).

65. Vincent, $\quad$ F. $\quad$ B., $\quad$ Bourke, P., Morand, E. $\quad$ F., Mackay, F. \& Bossingham, D. Focus on systemic lupus erythematosus in Indigenous Australians: towards a better understanding of autoimmune diseases. Intern. Med. J. 43, $227-$ 234 (2013).

66. Borchers, A. T., Naguwa, S. M., Shoenfeld, Y. \& Gershwin, M. E. The geoepidemiology of systemic lupus erythematosus. Autoimmun. Rev. 9, A277-A287 (2010).

67. Thumboo, J. et al. A comparative study of the clinical manifestations of systemic lupus erythematosus in Caucasians in Rochester, Minnesota, and Chinese in Singapore, from 1980 to 1992. Arthritis Rheum. 45, 494-500 (2001).

68. Golder, V., Connelly, K., Staples, M., Morand, E. \& Hoi, A. Association of Asian ethnicity with disease activity in SLE: an observational study from the Monash Lupus Clinic. Lupus 22, 1425-1430 (2013).

69. Ritterhouse, L. L. et al. B lymphocyte stimulator levels in systemic lupus erythematosus: higher circulating levels in African American patients and increased production after influenza vaccination in patients with low baseline levels. Arthritis Rheum. 63, 3931-3941 (2011). 
70. Kawasaki, A., Tsuchiya, N., Fukazawa, T., Hashimoto, H. \& Tokunaga, K. Analysis on the association of human BLYS (BAFF, TNFSF13B) polymorphisms with systemic lupus erythematosus and rheumatoid arthritis. Genes Immun. 3, 424-429 (2002).

71. Eilertsen, G. O., Van Ghelue, M., Strand, H. \& Nossent, J. C. Increased levels of BAFF in patients with systemic lupus erythematosus are associated with acute-phase reactants, independent of BAFF genetics: a case-control study. Rheumatology 50, 2197-2205 (2011).

72. Zayed, R. A. et al. B-cell activating factor promoter polymorphisms in Egyptian patients with systemic lupus erythematosus. Ann. Clin. Lab. Sci. 43, 289-294 (2013).

73. Koyama, T. et al. A novel polymorphism of the human APRIL gene is associated with systemic lupus erythematosus. Rheumatology (Oxford) 42, 980-985 (2003).

74. Lee, Y. H., Ota, F., Kim-Howard, X., Kaufman, K. M. \& Nath, S. K. APRIL polymorphism and systemic lupus erythematosus (SLE) susceptibility. Rheumatology (Oxford) 46, 1274 1276 (2007).

75. Kawasaki, A. et al. Role of APRIL (TNFSF13) polymorphisms in the susceptibility to systemic lupus erythematosus in Japanese. Rheumatology (Oxford) 46, 776-782 (2007).

76. Furuya, T., Koga, M., Hikami, K., Kawasaki, A. \& Tsuchiya, N. Effects of APRIL (TNFSF13) polymorphisms and splicing isoforms on the secretion of soluble APRIL. Mod. Rheumatol. 22, 541-549 (2012).

77. Jiang, C., Loo, W. M., Greenley, E. J., Tung, K. S. \& Erickson, L. D. B cell maturation antigen deficiency exacerbates lymphoproliferation and autoimmunity in murine lupus. J. Immunol. 186, 6136-6147 (2011).

78. Jacob, C. O. et al. Development of systemic lupus erythematosus in NZM 2328 mice in the absence of any single BAFF receptor. Arthritis Rheum. 65, 1043-1054 (2013).

79. Groom, J. R. et al. BAFF and MyD88 signals promote a lupus-like disease independent of T cells. J. Exp. Med. 204, 1959-1971 (2007).

80. Thangarajh,M.,Masterman,T.,Hillert,J., Moerk, S. \& Jonsson, R. A proliferation-inducing ligand (APRIL) is expressed by astrocytes and is increased in multiple sclerosis. Scand. J. Immunol. 65, 92-98 (2007).

81. George-Chandy, A., Trysberg, E. \& Eriksson, K. Raised intrathecal levels of APRIL and BAFF in patients with systemic lupus erythematosus: relationship to neuropsychiatric symptoms. Arthritis Res. Ther. 10, R97 (2008).

82. Bennett, L. et al. Interferon and granulopoiesis signatures in systemic lupus erythematosus blood. J. Exp. Med. 197, 711-723 (2003).

83. Peterson, K. S. et al. Characterization of heterogeneity in the molecular pathogenesis of lupus nephritis from transcriptional profiles of laser-captured glomeruli. J. Clin. Invest. 113, 1722-1733 (2004). 
84. Ytterberg, S. R. \& Schnitzer, T. J. Serum interferon levels in patients with systemic lupus erythematosus. Arthritis Rheum. 25, 401-406 (1982).

85. Kim, T. et al. Serum levels of interferons in patients with systemic lupus erythematosus. Clin. Exp. Immunol. 70, 562-569 (1987).

86. Rönnblom, L. Potential role of IFNalpha in adult lupus. Arthritis research \& therapy 12 (Suppl. 1), S3 (2010).

87. Lovgren, T., Eloranta, M. L., Bave, U., Alm, G. V. \& Ronnblom, L. Induction of interferon- $\alpha$ production in plasmacytoid dendritic cells by immune complexes containing nucleic acid released by necrotic or late apoptotic cells and lupus IgG. Arthritis Rheum. 50, 1861-1872 (2004).

88. Vallin, H., Blomberg, S., Alm, G. V., Cederblad, B. \& Rönnblom, L. Patients with systemic lupus erythematosus (SLE) have a circulating inducer of interferon- $\alpha$ (IFN- $\alpha$ ) production acting on leucocytes resembling immature dendritic cells. Clin. Exp. Immunol. 115, 196-202 (1999).

89. Panchanathan, R. \& Choubey, D. Murine BAFF expression is up-regulated by estrogen and interferons: implications for sex bias in the development of autoimmunity. Mol. Immunol. 53, $15-23$ (2013).

90. Litinskiy, M. B. et al. DCs induce CD40- independent immunoglobulin class switching through BLyS and APRIL. Nat. Immunol. 3, 822-829 (2002).

91. Harigai, M. et al. Excessive production of IFN- gamma in patients with systemic lupus erythematosus and its contribution to induction of B lymphocyte stimulator/B cell-activating factor/TNF ligand superfamily-13B. J. Immunol. 181, 2211-2219 (2008).

92. Yao, Y. et al. Neutralization of interferon-alpha/ beta-inducible genes and downstream effect in a phase I trial of an anti-interferon-alpha monoclonal antibody in systemic lupus erythematosus. Arthritis Rheum. 60, 1785-1796 (2009).

93. Jacob, N. et al. B Cell and BAFF dependence of IFN- $\alpha$-exaggerated disease in systemic lupus $\begin{array}{llll}\text { erythematosus-prone } & \text { NZM mice. }\end{array}$ J. Immunol. 186, 4984-4993 (2011).

94. Blanco, P., Palucka, A. K., Gill, M., Pascual, V. \& Banchereau, J. Induction of dendritic cell differentiation by IFN-alpha in systemic lupus erythematosus. Science 294, 1540-1543 (2001).

95. Joo, H. et al. Serum from patients with SLE instructs monocytes to promote $\operatorname{IgG}$ and $\operatorname{IgA}$ plasmablast differentiation. J. Exp. Med. 209, 1335-1348 (2012).

96. Kikly, K., Manetta, J., Smith, H. \& Wierda, D. Characterization of LY2127399, A neutralizing antibody for BAFF [abstract]. Arthritis Rheum. 60 (Suppl. 10), 693 (2009). 
97. Hsu, H. et al. A novel modality of BAFF-specific inhibitor AMG623 peptibody reduces B-cell number and improves outcomes in murine models of autoimmune disease. Clin. Exp. Rheumatol. 30, 197-201 (2012).

98. Wallweber, H. J., Compaan, D. M., Starovasnik, M. A. \& Hymowitz, S. G. The crystal structure of a proliferation-inducing ligand, APRIL. J. Mol. Biol. 343, 283-290 (2004).

99. Puga, I. et al. B cell-helper neutrophils stimulate the diversification and production of immunoglobulin in the marginal zone of the spleen. Nat. Immunol. 13, 170-180 (2012).

100. Holden, N. J. et al. ANCA-stimulated neutrophils release BLyS and promote B cell survival:

a clinically relevant cellular process. Ann. Rheum. Dis. 70, 2229-2233 (2011).

101. Assi, L. K. et al. Tumor necrosis factor- $\alpha$ activates release of B lymphocyte stimulator by neutrophils infiltrating the rheumatoid joint. Arthritis Rheum. 56, 1776-1786 (2007).

102. Suzuki, K. et al. Effect of interleukin-2 on synthesis of B cell activating factor belonging to the tumor necrosis factor family (BAFF) in human peripheral blood mononuclear cells. Cytokine 44, 44-48 (2008).

103. Zhang, W. et al. hsBAFF enhances activity of NK cells by regulation of CD4(+) T lymphocyte function. Immunol. Lett. 120, 96-102 (2008). 


\section{Figure 1. Soluble BAFF and APRIL signalling.}

BAFF and APRIL are type II transmembrane proteins, but BAFF can be processed into a soluble cytokine after cleavage at a furin protease site. APRIL is soluble, having been cleaved intracellularly. BAFF only has weak affinity for BCMA. BAFF-R is essential for survival and maturation of immature B cells. TACI is critical for T-cell-independent responses of B cells to type I and type II antigens, negative regulation of the size of the B-cell compartment and class-switch recombination. BCMA promotes plasma-cell survival. Dashed line indicates that BAFF possibly binds $\mathrm{NgR}$, which is expressed on neurons and astrocytes, and has been shown to mediate negative effects on neuron outgrowth. Abbreviations: APRIL, a proliferation-inducing ligand (also known as TNF ligand superfamily member 13); BAFF, Bcell-activating factor of the TNF family (also known as TNF ligand superfamily member 13B); BAFF-R, BAFF receptor (also known as TNF receptor superfamily member 13C); BCMA, B-cell maturation antigen (also known as TNF receptor superfamily member 17); $\mathrm{NgR}$, Nogo-66 receptor (also known as reticulon 4 receptor); TACI, transmembrane activator and cyclophilin ligand interactor (also known as TNF receptor superfamily member 13B).

\section{Figure 2. Structural variants of BAFF and APRIL.}

Different BAFF-APRIL structures might be involved in the pathogenesis of SLE, or might counteract the effects of BAFF and APRIL homotrimers by sequestering monomeric components required for their formation, competing for receptor binding or inducing suboptimal signals. APRIL interacts with polysaccharide side chains of HSPG, and interactions

of TACI and HSPG regulate antibody class-switching. Solid lines indicate known interactions. Dashed lines indicate hypothetical interactions. Abbreviations: APRIL, a proliferation-inducing ligand (also known as TNF ligand superfamily member 13); BAFF, Bcell-activating factor of the TNF family (also known as TNF ligand superfamily member 13B); BAFF-R, BAFF receptor (also known as TNF receptor superfamily member 13C); BCMA, B-cell maturation antigen (also known as TNF receptor superfamily member 17); HSPG, heparan sulphate proteoglycans; SLE, systemic lupus erythematosus; TACI, transmembrane activator and cyclophilin ligand interactor (also known as TNF receptor superfamily member 13B); TWEAK, TNF-related weak inducer of apoptosis (also known as 
TNF ligand superfamily member 12); TWE-PRIL; a hybrid of TWEAK and APRIL.

\section{Figure 3. Role of BAFF in the pathogenesis of SLE.}

$\mathrm{n}$ SLE, it is believed that antibody-nucleic acid immune complexes (1), for example ssRNA or DNA from dead cells, are bound by Fc $\gamma$ RIIa, activating TLRs and IFN- $\alpha$ production (2). IFN- $\alpha$ increases BAFF production (3). BAFF interacts with receptors on B cells (4). Excess BAFF can increase autoreactive B-cell survival, driving autoimmunity (5). TLR4 and TACI signalling cooperate to commit MZ B cells to apoptosis via induction of Fas and FasL (6), possibly contributing to the mechanism that terminates the short-lived antibody response of activated innate $\mathrm{B}$ cells. This mechanism is defective in BAFF-overexpressing transgenic mice (7; Box 1). Abbreviations: BAFF, B-cell-activating factor of the TNF family (also known as TNF ligand superfamily member 13B); BAFF-R, BAFF receptor (also known as TNF receptor superfamily member 13C); BCMA, B-cell maturation antigen (also known as TNF receptor superfamily member 17); DC, dendritic cell; FasL, Fas ligand; Fc $\gamma$ RIIa, immunoglobulin $\gamma \mathrm{Fc}$ region receptor IIa; LPS, lipopolysaccharide; MyD88, myeloid differentiation primary response protein MyD88; MZ, marginal zone; ssRNA, single-stranded RNA; TACI, transmembrane activator and cyclophilin ligand interactor (also known as TNF receptor superfamily member 13B); TLR4, Toll-like receptor 4.

\section{Competing Interests}

F.M. and E.F.M. declare that they have acted as consultants for Eli Lilly and GSK. P.S. declares that he has a research agreement with Merck-Serono. F.B.V. declares no competing interests.

\section{Author contributions}

All authors researched the data for the article. F.B.V. wrote the manuscript and all authors reviewed/edited the manuscript before submission. 


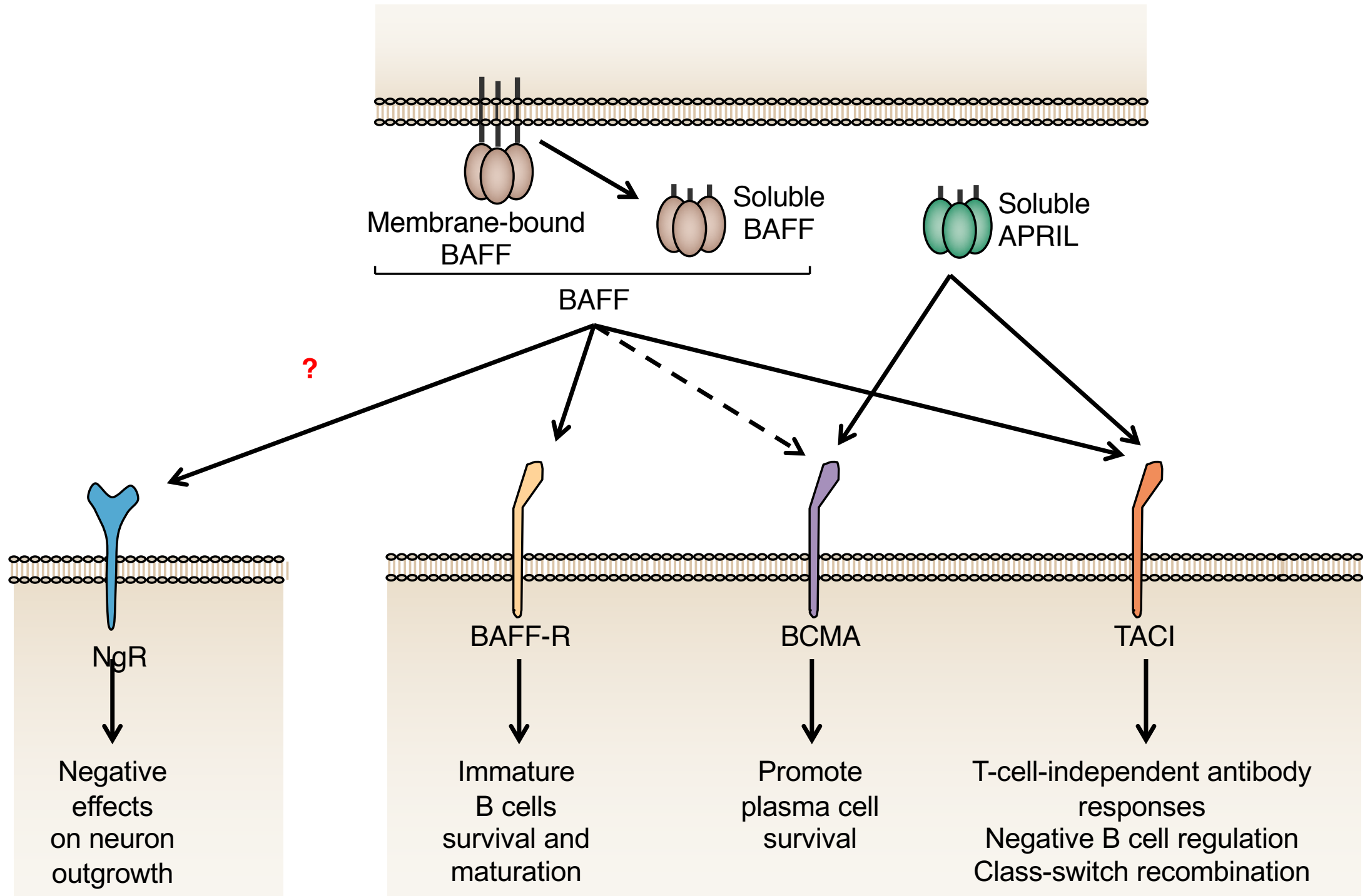

Neurons and astrocytes

Primarily expressed on B cells, plasmablasts and plasma cells 


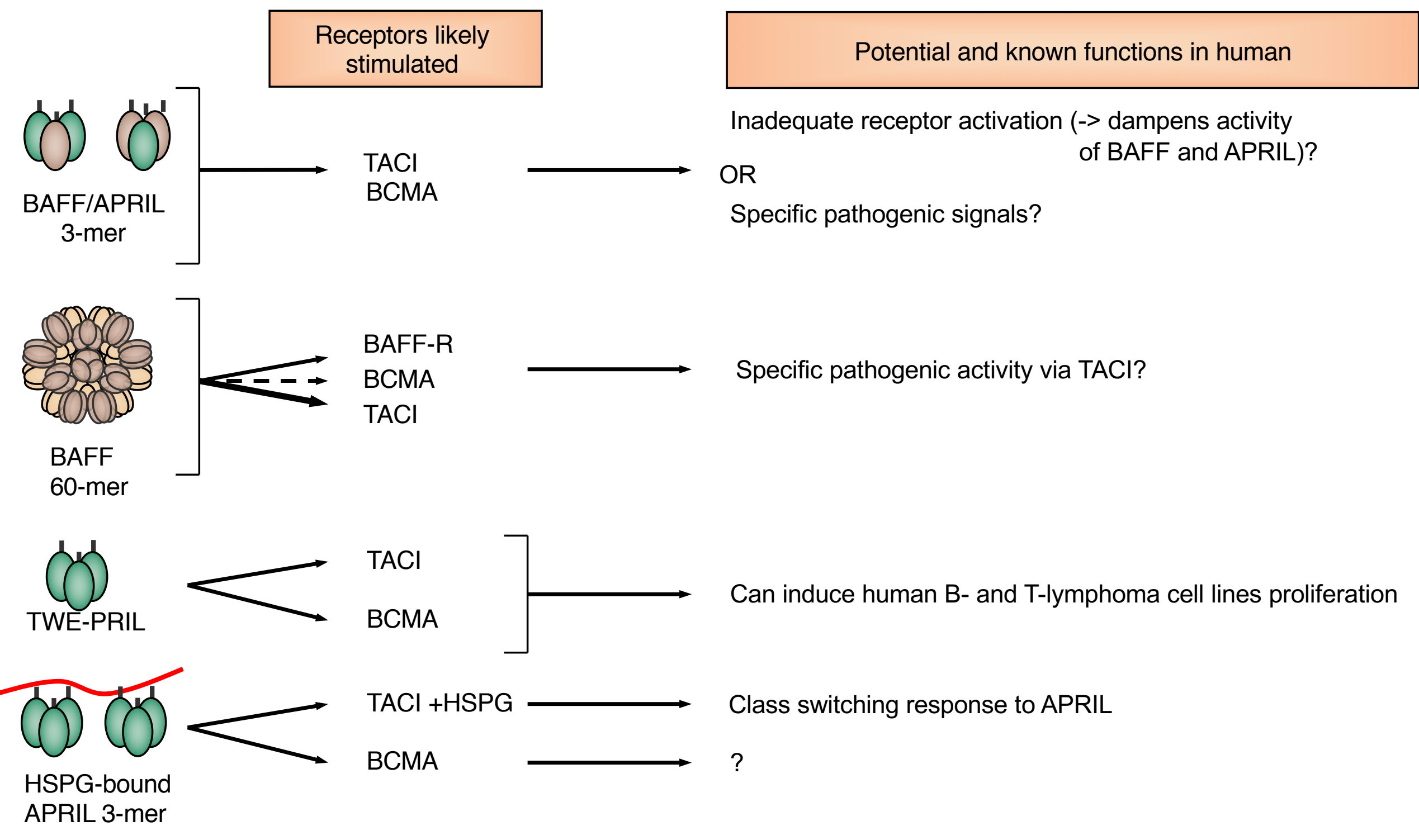


Necrotic/Apoptotic cells

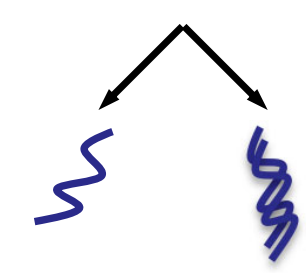

SsRNA DNA

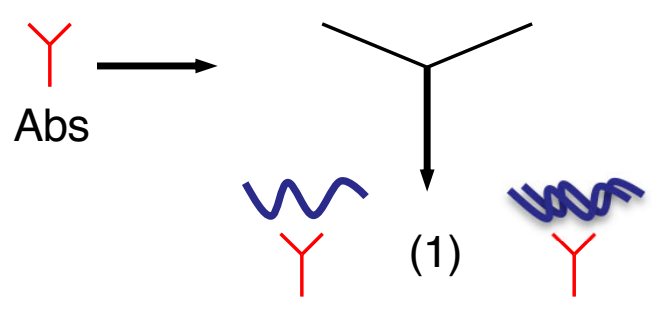

Ab-nucleic acid immune complexes

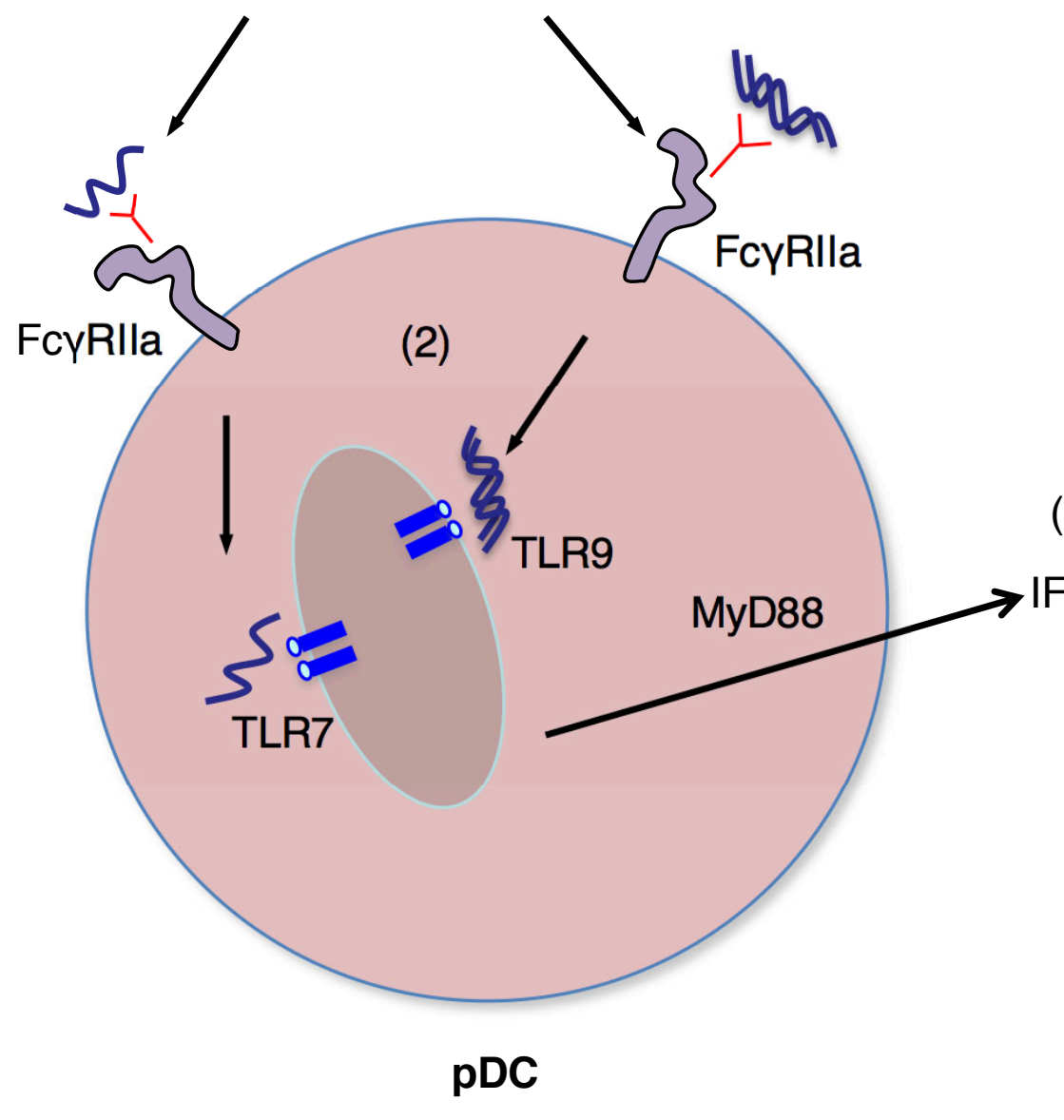

Autoreactive B cells, pathogenic antibodies

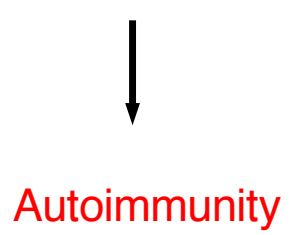

(3) IFN- $\alpha$
(5)

B cell

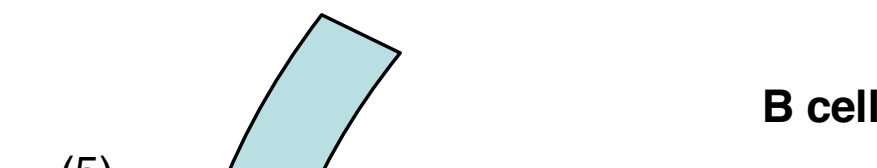

(4)

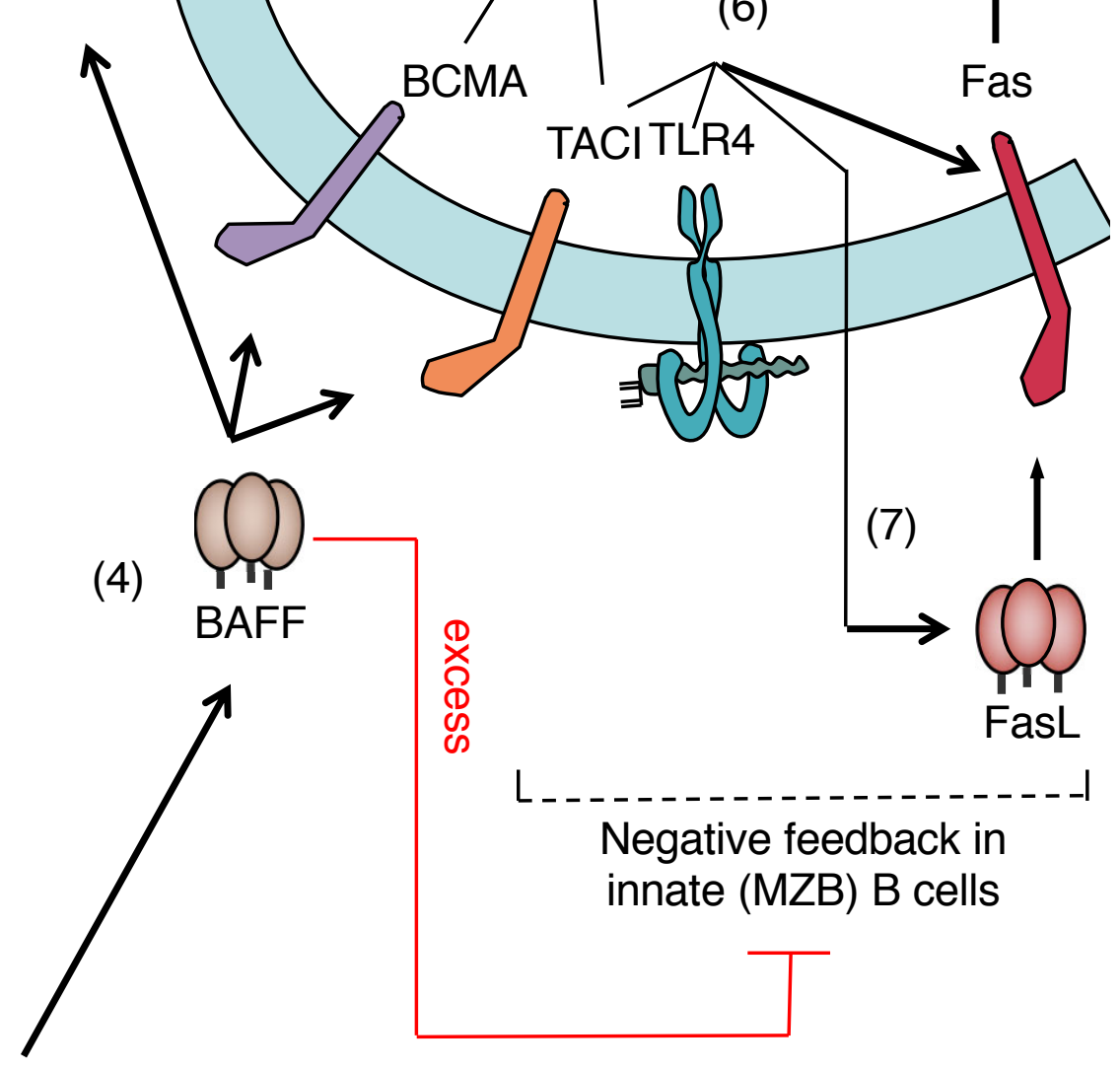

Cell death

(6) 